\title{
Higher-order structures of HIV Integrase: Drug-induced Aggregates of HIV Integrase are Weak Gels
}

\author{
Kushol Gupta ${ }^{1}$, Grant Eilers ${ }^{2}$, Audrey Allen², Carolina Giraldo ${ }^{1}$, Bob Sharp ${ }^{1}$, Young Hwang ${ }^{2}$, \\ Katrina $\mathrm{Cruz}^{3}$, Paul Jamney ${ }^{3}$, Frederic D. Bushman ${ }^{2+}$, and Gregory D. Van Duyne ${ }^{1+}$ \\ Email Contact: kgupta@pennmedicine.upenn.edu \\ From the Perelman School of Medicine, University of Pennsylvania. ${ }^{1}$ Department of \\ Biochemistry and Biophysics 242 Anatomy-Chemistry Building Philadelphia, PA, 19104-6059 \\ U.S.A. ${ }^{2}$ Department of Microbiology 3610 Hamilton Walk Philadelphia, PA 19104-6076 U.S.A. \\ ${ }^{3}$ Department of Bioengineering and Departments of Physiology and Physics and Institute for \\ Medicine and Engineering, University of Pennsylvania, Philadelphia, PA, 19104, USA
}

Effective antiviral agents are available for treating HIV, but the virus commonly evolves to become resistant to inhibitors-thus interest continues in developing new antiviral agents. Effective inhibitors have been developed that target the active site of HIV integrase (IN). We have been studying another class of small molecules, the allosteric inhibitors of integrase (ALLINIs). The major effect of ALLINIs is observed during virion maturation, where ALLINI treatment results in IN aggregation and the formation of aberrant particles. We previously determined the structure of full-length HIV IN bound with an ALLINI at 4.4 $\AA$ resolution. We have extended these studies by analyzing another ALLINI, BI-D, and two additional forms of IN that are found in replication-competent viruses $\left(\mathrm{IN}^{\mathrm{W} 131 \mathrm{C}}\right.$ and $\left.\mathrm{IN}^{\mathrm{N} 222 \mathrm{~K}}\right)$. Together these structures define the structural features of the ALLINI-mediated interactions that initiate aggregation. We sought to develop a more complete view of the structural changes mediating drug-induced aggregation by applying multiple biophysical approaches. Using size-exclusion chromatography in-line with synchrotron small angle X-ray scattering (SEC-SAXS) with evolving factor analysissingular value decomposition (EFA-SVD), we computationally decomposed, characterized, and modeled the oligomers formed by IN in solution. Comparisons with SAXS/SANS (small-angle Xray/neutron scattering) contrast variation analyses of reconstituted IN-DNA complexes indicate that the IN tetramers formed in the absence of DNA are distinct to those formed within the intasome. Using SANS, scanning electron microscopy (SEM), and rheology, we find that the higher-order aggregates induced by the prototype ALLINIs BI-D and BI-224436 have the characteristics of weak 3D-gels. Gel formation is inhibited by the presence of the host factors LEDGF/p75 and TNPO3, as well as resistance mutations. We further delineate the contribution of catalytic core domain (CCD) and C-terminal domain (CTD) interactions to gel formation. Our results suggest a novel structural model for IN oligomerization and drug-induced aggregation involving formation of branched polymers. Characterization of the higher order IN-ALLINI complexes and resulting escape mutants provides data useful in optimizing ALLINI drug design and understanding mechanisms of resistance. 